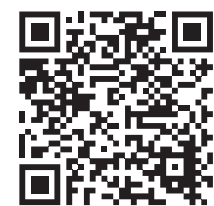

* Profesor y Médico del Centro de Investigación Educativa y Formación Docente. Centro de Investigación Educativa y Formación Docente. * Médica Especialista en Medicina de Urgencias del Hospital General de Zona Núm. 1-A «Dr. Rodolfo Antonio de Mucha Macías». Servicio de Urgencias. § Directora de la Clínica de Columna Vertebral, Guadalajara Jalisco.

Correspondencia: FHP, mesias.francisco @gmail.com Conflicto de intereses: Los autores declaramos no tener ningún conflicto de intereses, no recibimos aportaciones de organizaciones o empresas internas o externas. Los autores declaramos que no tenemos ninguna relación de parentesco o económica para con los voluntarios.

Citar como: Hernández PF, Vargas PEI, Tello RMR. Creencias sobre la pandemia y las medidas de protección en pacientes que acuden al servicio de urgencias por probable COVID-19. Rev CONAMED. 2021; 26(3): 134-142. https:// dx.doi.org/10.35366/101678

\section{Creencias sobre la pandemia y las medidas de protección en pacientes que acuden al servicio de urgencias por probable COVID-19}

\author{
Beliefs about pandemic and protection measures in patients \\ going to the emergency department for suggestive COVID-19 \\ Francisco Hernández Pérez,* Erika Itzel Vargas Palma,‡María del Refugio Tello Ramírez ${ }^{\S}$
}

\begin{abstract}
RESUMEN
En México los casos positivos para SARS-CoV-2 son altos, con casi dos millones de contagiados y cerca de 150,000 fallecidos, en lo que se denomina la «segunda ola». Una de las explicaciones de tal fenómeno es la falta de apego a las medidas sanitarias que se relacionan estrechamente con la cultura y las creencias. Objetivo: Identificar las creencias más frecuentes sobre la pandemia y las medidas de protección de los pacientes que acuden al servicio de urgencias por probable COVID-19. Material y métodos: Se realizó un estudio trasversal analítico. Se invitó a 100 pacientes que acudieron a la unidad de triaje respiratorio por referir síntomas sugestivos de COVID-19 de un hospital de segundo nivel de atención. Se excluyó a los pacientes con asistencia ventilatoria o graves. Se realizó una entrevista de 12 preguntas de respuesta dicotómica y se reportaron como porcentajes. Resultados: Se entrevistó a 100 voluntarios con datos sugestivos de COVID-19. La edad media fue de $50.96 \pm 14.9$ años, 54\% $(n=54)$ eran hombres con un nivel educativo superior a secundaria en 52\%; de los encuestados, 33\% refirieron haber sido contagiados por un familiar, $24 \%$ aceptaron que no creían en la existencia del virus del SARS-CoV-2 antes de infectarse (porcentaje que incrementaba a $44.8 \%$ en hombres con secundaria o menos), 10\% no usaba cubrebocas. Los hombres creían menos en la existencia del virus (21 de 54) ( $p=$ 0.003), $43.75 \%$ de los voluntarios con un nivel educativo de secundaria o menos creían que en los hospitales se les extraía líquido de las rodillas (21 de 48) ( $p=0.013)$. Conclusiones: En el presente trabajo un cuarto de los encuestados no creía en la existencia del virus SARS-CoV-2, el cual se incrementaba en los hombres voluntarios de nivel educativo bajo. Asimismo, la mayoría no estaban vacunados contra la influenza y un tercio tenía la creencia de que se les extraía líquido de la rodilla a los pacientes y se les dejaba morir en los hospitales.
\end{abstract}

Palabras clave: Creencias, COVID-19, pandemia

\begin{abstract}
In Mexico, the positive cases for SARS-CoV-2 is high, with almost two million infected and about 150 thousand deaths, in what is called the «second wave». One of the explanations for this phenomenon is the lack of adherence to sanitary measures that are closely related to culture and beliefs. Objective: To identify the most frequent beliefs about the pandemic and the protection measures of patients who come to the emergency department due to probable COVID-19. Material and methods: An analytical cross-sectional study was carried out. One hundred patients who came to the respiratory triage unit for referring symptoms suggestive of COVID-19 from a second-level hospital were invited. Patients with ventilatory assistance or severely ill were excluded. An interview of 12 dichotomous answer questions was conducted and they were reported as percentages. Results: One hundred volunteers were interviewed with data suggestive of COVID-19. The mean age was $50.96 \pm 14.9$ years, $54 \%(n=54)$ were men with an educational level higher than secondary in $52 \%$. Thirty-three respondents reported having been infected by a relative. $24 \%$ of the interviewees accepted that they did not believe
\end{abstract}


Financiamiento: El financiamiento corrió a cargo de los investigadores.

Recibido: 21/01/2021. Aceptado: 26/08/2021. in the existence of the SARS-CoV-2 virus before becoming infected (it increased to $44.8 \%$ in men with secondary school or less), $10 \%$ did not use mouthwash. Men believed less in the existence of the virus (21 of 54) ( $p=0.003)$. 43.75\% of the volunteers with a high school education level or less believed that the hospitals extracted fluid from their knees (21 of 48) ( $p=0.013)$. Conclusions: In the present study, a quarter of the respondents did not believe in the existence of the SARS-CoV-2 virus and it increased with male volunteers of low educational level, likewise, most were not vaccinated against influenza and a third believed that fluid was taken from patients' knees and left to die in hospitals.

Keywords: Beliefs, COVID-19, pandemic.

\section{INTRODUCCIÓN}

El 31 de diciembre de 2019 China informó de los primeros casos de una nueva enfermedad respiratoria en humanos que apareció primero en Wuhan, Provincia de Hubei y que involucró a un nuevo coronavirus (SARS-CoV-2).'Para el 11 de marzo de 2020 la Organización Mundial de la Salud (OMS) declaró la enfermedad como pandemia por coronavirus 2019 (COVID-19). ${ }^{2}$

En México el primer caso reportado fue el 28 de febrero y la primera muerte el 19 de marzo, para el 23 de marzo las autoridades declararon al COVID-19 como una emergencia de salud, comenzando el aislamiento social como la principal acción para contener la epidemia. La fase de aceleración en México se declaró casi un mes más tarde, el 21 de abril, cuando el número de casos confirmados fue de 9,501 y 857 muertes. ${ }^{3}$ El impacto no sólo fue en el sistema de salud mundial, sino político, cultural y por supuesto económico. ${ }^{4}$

En el reporte diario del COANCYT hasta mediados de enero de 2021, el número de contagiados a nivel mundial sobrepasaba 96,200,000 y más de 2,000,000 de fallecidos, en México en esta misma época el número era de 1,700,000 de positivos acumulados y más de 125,000 fallecidos, con un incremento ponderal en la última quincena de entre 0.3 y $1.3 \%$, es decir, entre 10,000 y 20,000 nuevos casos diarios, con un número de fallecidos entre 500 y 1,400 fallecidos al día, ${ }^{5}$ a pesar de que desde inicios de la pandemia el gobierno mexicano impuso un modelo de semaforización (rojo, anaranjado, amarillo y verde) según el grado de riesgo de contagio de la población y que fue aplaudido por la propia OMS. ${ }^{6}$

\section{Marco teórico}

Pero, más allá del origen del virus y su potencial tratamiento médico y/o la generación de la vacuna, su adquisición, proceso y tratamiento es de principio a fin un proceso psicológico. En ese sentido, las investigaciones posteriores han generado abundante evidencia que muestra que tanto la prevención, la adherencia al tratamiento o el enfrentamiento y avance de múltiples enfermedades son un tema de comportamiento. La evidencia muestra que los mecanismos por los cuales adquirimos, mantenemos y eliminamos nuestros comportamientos son principalmente por medio del aprendizaje. De esta forma, el pasar de una condición de ausencia de enfermedad a estar enfermo, e incluso el éxito o fracaso del tratamiento, estará determinado por nuestras creencias (cogniciones), emociones (como predisposiciones a la acción) y finalmente nuestro comportamiento en pro o en riesgo para la salud. Una manifestación extrema es el llamado «optimismo ilusorio», que lleva a que los individuos esperen cosas positivas casi de manera mágica independientemente de lo que ellos hagan. Este último caso se ha identificado como riesgoso para la salud, ya que podría llevar a que sujetos con esa tendencia pseudoptimista e ilusoria tengan incluso comportamientos riesgosos para su salud, ya que esperan que ellos no se enfermarán. En pocas palabras, cuando los individuos perciben que entre la emisión de una determinada conducta y la «eventual» llegada de las consecuencias (sean positivas o negativas), es poco probable o pase mucho tiempo para que este comportamiento se adquiera o modifique. El gran problema de esta enfermedad ha estado vinculado al comportamiento de los individuos y a la necesidad de cambios en el estilo de vida habitual, y este comportamiento está estrechamente ligado a nuestras cogniciones, emociones y factores sociales y culturales. ${ }^{7,8}$

Estudios sobre la pandemia por COVID-19 han revelado que las personas tienden a percibir mayores riesgos para la salud cuando envejecen, 
ya que aumenta el riesgo para su salud y que las variables que representan el estatus social como la educación y los ingresos suelen asociarse positivamente con percepciones de riesgo, lo que sugiere que la educación superior y los ingresos conducen a un nivel más alto de percepción de riesgo, $910 \mathrm{Un}$ buen ejemplo de ambas hipótesis es el personal de salud que ha visto modificados drásticamente sus patrones de socialización por las condiciones a las que han sido sometidos.1-13

Este año el CONACYT realizó un estudio sobre creencias y encontró que las expresiones que mayor porcentaje obtuvieron podrían ubicarse en percepciones que empatan con las perspectivas científicas, naturalistas y conspiracionistas, mientras que las asociadas a una perspectiva religiosa tienen los porcentajes más bajos..$^{14}$ Semejantes resultados fueron encontrados por Mejía y colaboradores. ${ }^{15}$

Por otro lado, los medios de comunicación han tenido un papel preponderante y muchos de ellos han facilitado la difusión de desinformación de COVID-19, ya sea al maximizar o minimizar el riesgo de esta enfermedad en comparación con las demás.9,16 En el estudio Regí y su equipo, los encuestados percibieron que había mucha publicidad mediática, es decir, una influencia repentina de conocimiento a través de diferentes medios (infodemia) que generan confusión entre el público con respecto a los cambios de comportamiento. ${ }^{8}$ En el estudio de Gohel y colegas se reporta que los encuestados dijeron que sus principales fuentes de información fueron las redes sociales (Facebook, WhatsApp, YouTube, Instagram) (17\%) seguidas de los medios informativos (TV/vídeo) (20.84\%), es decir, la mayoría de los encuestados obtuvo conocimiento sobre COVID-19 de las redes sociales (65.17\%) y tristemente sólo 37.8 y $29.7 \%$ de las personas entrevistadas de EUA y el Reino Unido respectivamente creían que usar cubrebocas podría proteger a las personas de infectarse con COVID-19. ${ }^{17}$ Por otro lado, Raude y colaboradores reportan que cerca de la mitad de los encuestados estiman que era menos probable que la infección les ocurriera a ellos que a la población general. Para los autores, la mayoría de las personas en Europa estaban más sujetas a un optimismo «poco realista» sobre el riesgo de infección por coronavirus. ${ }^{18}$

La vacuna merece una mención aparte. Desde hace tiempo se sabe que la aplicación de vacunas está relacionada con mitos, creencias filosóficas o religiosas, rumores e información que no se sustentan en evidencia científica y que se ve empoderada por el surgimiento de los movimientos antivacunas que han provocado, en parte, el resurgimiento de enfermedades que, como el sarampión, estaban en vías de erradicación. ${ }^{19}$ Fisher reporta que sólo $57.6 \%$ de los participantes en la encuesta tenían intención de vacunarse contra el coronavirus, 31.6\% (n: 313) no estaban seguros y 10.8\% (n: 107) no tenían la intención de vacunarse. Los factores asociados de manera independiente con la indecisión a la vacuna incluyeron edad más joven (< 60 años), raza negra, menor nivel educativo y no haber recibido la vacuna contra la influenza en el año anterior. ${ }^{20}$

Finalmente, habrá que reconocer que todos podemos ser portadores, contagiar y ser contagiados, y que el curso y pronóstico aún es incierto. La necesidad de mantener distanciamiento físico, dentro y fuera del domicilio conlleva que la idea de cumplimiento no siempre sea aceptada y mucho menos se lleve a cabo por una parte de la población. ${ }^{21} \mathrm{Al}$ parecer «los grupos sociales con más poder económico y mayor nivel de educación se enfrentan muchísimo mejor a la crisis sanitaria. Las desigualdades sociales en salud están ahí y los de menores ingresos y más creencias ilusorias son los más vulnerables. Esas desigualdades tienen que ver en un alto grado con el tipo de políticas sociales y económicas que se aplican en cada país. ${ }^{16}$ Sin embargo, se desconocen tantas cosas de la pandemia que es difícil prever un resultado final aun con la obtención de la vacuna, pero lo cierto es que las creencias afectan de una manera importante no sólo la percepción de esta alerta sanitaria, sino la forma en que se actúa. En ese sentido, nuestro trabajo se enfoca en esas creencias y su efecto en la prevalencia de la enfermedad.

\section{MATERIAL Y MÉTODOS}

Se realizó un estudio trasversal analítico. Se invitó a todos los pacientes que acudían a la unidad de triaje respiratorio por referir síntomas sugestivos de COVID-19 al Servicio de Urgencias (triaje respiratorio) del Hospital de Mucho Macías (HCZ 1-A) del Instituto Mexicano del Seguro Social. Se excluyó a los pacientes con asistencia ventilatoria o graves. Se realizó una entrevista de 12 preguntas de respuesta 
dicotómica sobre sus creencias en cuanto a la existencia de la enfermedad así como las medidas de protección, contagio y percepción de la acción del gobierno y medios de comunicación. Se reportaron como porcentajes.

\section{RESULTADOS}

Se entrevistó a 100 pacientes que aceptaron participar en el estudio y que tenían síntomas de probable COVID-19. Los voluntarios fueron abordados en la unidad de triaje respiratorio. La edad media fue de $50.96 \pm 14.9$ años (rango de 21 a 81 años, $70 \%$ era mayor de 40 años), con una distribución normal, $54 \%(n=54)$ eran hombres con un nivel educativo superior a la secundaria en $52 \%$, estado civil en su mayoría casados (45\%). De los encuestados, 33 refirieron haber sido contagiados por un familiar, un hijo y su pareja (siete y cinco encuestados respectivamente) (Tab/a 7).

En cuanto a la encuesta de opinión, 24\% de los entrevistados aceptó que no creía en la existencia del virus del SARS-CoV-2 antes de infectarse, porcentaje que se incrementó a 53\% cuando se les preguntó si algún familiar no creía en la existencia del virus. De los encuestados, 10\% no usaba crubebocas y 73\% refirió tener a un familiar enfermo de COVID-19 (Tabla 2).

Al compararse por sexo, sólo se encontró que los hombres creían menos en la existencia del virus (21 de 54), habiendo una diferencia significativa con las mujeres (sólo tres de 43) ( $p=0.003)$. En el resto de los valores no se encontró diferencia significativa (Tabla 3) excepto en la factibilidad de la «contagio de rebaño» como se retoma en la Tabla 4.

Cuando se analizó por edad, entre aquéllos con 40 años o menos comparados con mayores de 41 años o más, no se encontró ninguna diferencia significativa en sus respuestas ( $p>0.05)$ Y al comparar nivel educativo, voluntarios de ambos sexos, con un nivel educativo de secundaria o menor, se encontró que $43.75 \%$ de los que tenían un nivel educativo bajo (secundaria o menos) sí creían que en los hospitales se les extraía líquido de la rodilla a los pacientes (21 de 48), ( $p=0.013)$. En el resto de las respuestas no se encontró diferencia significativa $(p>0.05)$.

Al separar por sexo, los voluntarios hombres con nivel educativo bajo (secundaria o menos) se encontraron diferencias significativas sólo en la pregunta sobre la existencia del virus, ya que la mayoría de hombres con escasa instrucción no creían en la existencia del virus (44.8\%), habiendo

\begin{tabular}{|c|c|}
\hline \multicolumn{2}{|c|}{$\begin{array}{l}\text { Tabla 1: Características generales } \\
\text { de los voluntarios } N=100 .\end{array}$} \\
\hline Característica & $\mathrm{n}=\%$ \\
\hline Edad, años & $50.96 \pm 14.9$ \\
\hline \multicolumn{2}{|l|}{ Sexo } \\
\hline Masculino & 54 \\
\hline Femenino & 46 \\
\hline \multicolumn{2}{|l|}{ Estado civil } \\
\hline Soltero & 27 \\
\hline Casado & 45 \\
\hline Unión libre & 19 \\
\hline Divorciado & 3 \\
\hline Viudo & 6 \\
\hline \multicolumn{2}{|l|}{ Ocupación } \\
\hline Desempleado & 5 \\
\hline Hogar & 22 \\
\hline Comerciante & 21 \\
\hline Obrero & 3 \\
\hline Oficinista & 9 \\
\hline Personal de salud & 6 \\
\hline Taxista & 3 \\
\hline Técnico & 5 \\
\hline Otro & 19 \\
\hline \multicolumn{2}{|l|}{ Forma de contagio } \\
\hline No sabe & 17 \\
\hline Familiar & 33 \\
\hline En el transporte & 4 \\
\hline En su centro de trabajo & 34 \\
\hline En velatorio o sepelio & 3 \\
\hline En un viaje largo & 2 \\
\hline $\begin{array}{l}\text { En una reunión con } \\
\text { compañeros/amigos }\end{array}$ & 4 \\
\hline En el mercado o centro comercial & 4 \\
\hline \multicolumn{2}{|l|}{ Preferencia de vacuna } \\
\hline Ninguna & 17 \\
\hline Spunik V (Rusa) & 42 \\
\hline Inglesa & 12 \\
\hline Norteamericana (Moderna) & 24 \\
\hline Cualquiera & 5 \\
\hline
\end{tabular}


Tabla 2: Respuestas de todos los voluntarios.

\begin{tabular}{lcc}
\hline & & $\mathrm{n}=\%$ \\
\cline { 2 - 3 } Pregunta & Sí & No \\
\hline \multirow{2}{*}{ ¿Antes de enfermarse, usted creía en la existencia del virus de COVID-19? } & 76 & 24 \\
¿Antes de enfermarse, usted usaba cubrebocas? & 90 & 10 \\
¿Usted cree que el cubrebocas sirve? & 83 & 17 \\
¿Tuvo o tiene algún familiar enfermo de COVID-19? & 73 & 27 \\
¿Algún familiar no cree o no creía en el virus? & 54 & 46 \\
¿Cree que en algunos hospitales los dejen morir? & 36 & 64 \\
¿Cree que algunos médicos sí les extrajeron líquido de las rodillas a pacientes? & 32 & 68 \\
¿Está usted de acuerdo con la técnica de infección de «rebaño» para acabar más rápido & 9 & 91 \\
con la pandemia? & 80 & 20 \\
¿Cree que la vacuna sirva? & 40 & 60 \\
¿Está vacunado contra la influenza? & 77 & 23 \\
¿Cree que las medidas recomendadas por el gobierno sirvan de algo? & 37 & 63 \\
¿Cree que la información dada por los medios de comunicación es confiable? & \\
\hline
\end{tabular}

una diferencia significativa con los hombres con mayor nivel educativo ( $p=0.003)$ y al hacer la estimación de riesgo se observó que ser hombre con nivel educativo bajo, aumenta 1.7 veces el no creer en la existencia del virus (IC95\% 1.09-2.7).

Finalmente, al preguntar si estaban de acuerdo en la «técnica de contagio por rebaño» la mayoría de hombres con baja escolaridad estuvieron de acuerdo en dicha técnica, lo que fue estadísticamente significativo, como se muestra en la Tabla 4.

\section{DISCUSIÓN}

Nuestros resultados manifiestan un grave problema cultural lleno de creencias no fundamentadas alrededor de una pandemia que ya sobrepasó 2,000,000 de muertos a nivel global; casi un cuarto de los entrevistados negaba la existencia del virus SARS-CoV-2 previo a enfermarse, mismo que se incrementaba a más de la mitad (53\%) en un miembro de la familia que no creía en su existencia. Al parecer tiene relación con el sexo y el nivel académico, ya que en el hombre subía a $38 \%$ la no creencia en la existencia del virus y si ese hombre tenía una escolaridad de secundaria o menos subía a 44\%. Un tercio refirió haber sido contagiado por un familiar, pero 73\% refirió tener algún familiar enfermo.
Tres datos curiosos son que 32\% de los voluntarios creían que en los hospitales se les extraía líquido de las rodillas a los pacientes para comercializarla, un porcentaje similar (32\%) pensaba que en los hospitales se deja morir a los pacientes y la mayoría de los hombres con baja escolaridad sí consideraban la «técnica de contagio por rebaño» como una estrategia adecuada.

Estudios relacionados a las creencias reportan datos variados, así Asimakopoulou y colaboradores encontraron que la mayoría de voluntarios pensaba que no enfermaría o sería leve en comparación con personas de la misma edad y sexo. ${ }^{22}$ Y estudios sociales están de acuerdo en que durante las pandemias hay un incremento en las teorías pseudocientíficas como lo reportan Escola y su equipo. ${ }^{23}$ Mientras Sallam y colegas relacionan estas creencias conspirativas a un nivel académico bajo.

En cuanto al sexo, las mujeres (en nuestro estudio fueron hombres) y los participantes con ingresos más bajos tenían más probabilidades de creer que la enfermedad está relacionada con una conspiración. ${ }^{24}$ Y Freeman y colaboradores lo asociaron a una menor adherencia a las pautas gubernamentales así como menor predisposición a aplicarse la vacuna. ${ }^{25}$

Por su lado Panchal y Jack consideran que la creencia en teorías conspirativas se debe a 
condiciones ambientales particularesy psicológicas basadas en tres motivaciones psicológicas: epistémica, existencial y social. La motivación epistémica se relaciona con la de un individuo o comprensión y conocimiento del grupo de un fenómeno. Las teorías de la conspiración pueden permitir a los individuos preservar un sentido de comprensión ante la incertidumbre y contradicción. Es notorio que estas creencias se vuelven más fuertes cuando los eventos son generalizados y/o significativos. Las creencias

Tabla 3: Comparación de las respuestas por sexo.

\begin{tabular}{|c|c|c|c|}
\hline Pregunta & $\begin{array}{c}\text { Hombres } \\
\mathrm{n}(\%)\end{array}$ & $\begin{array}{c}\text { Mujeres } \\
\mathrm{n}(\%)\end{array}$ & $\mathrm{p}\left(\chi^{2}\right)$ \\
\hline \multicolumn{4}{|c|}{ ¿Antes de enfermarse, usted creía en la existencia del virus de COVID-19? } \\
\hline Sí & $33(61.1)$ & $43(93.4)$ & \multirow[t]{2}{*}{0.000} \\
\hline No & $21(38.9)$ & $3(6.7)$ & \\
\hline \multicolumn{4}{|c|}{ ¿Antes de enfermarse, usted usaba cubrebocas? } \\
\hline Sí & $47(87)$ & $43(93.4)$ & \multirow[t]{2}{*}{0.233} \\
\hline No & $7(13)$ & $3(6.7)$ & \\
\hline \multicolumn{4}{|c|}{ ¿Usted cree que el cubrebocas sirve? } \\
\hline Sí & $44(81.4)$ & $39(84.7)$ & \multirow[t]{2}{*}{0.434} \\
\hline No & $10(18.6)$ & $7(15.3)$ & \\
\hline \multicolumn{4}{|c|}{ ¿Tuvo o tiene algún familiar enfermo de COVID $19 ?$} \\
\hline Sí & $37(64.9)$ & $36(66.6)$ & \multirow[t]{2}{*}{0.193} \\
\hline No & $17(35.1)$ & $10(33.4)$ & \\
\hline \multicolumn{4}{|c|}{ ¿Algún familiar no cree o no creía en el virus? } \\
\hline Sí & $31(57.4)$ & $23(50)$ & \multirow[t]{2}{*}{0.295} \\
\hline No & $23(42.6)$ & $23(50)$ & \\
\hline \multicolumn{4}{|c|}{ ¿Cree que en algunos hospitales los dejen morir? } \\
\hline Sí & $20(37)$ & 16(65.2) & \multirow[t]{2}{*}{0.491} \\
\hline No & $34(63)$ & $30(34.8)$ & \\
\hline \multicolumn{4}{|c|}{ ¿Crees que algunos médicos sí les extrajeron líquido de las rodillas a pacientes? } \\
\hline Sí & $18(33.4)$ & $14(30.4)$ & \multirow[t]{2}{*}{0.463} \\
\hline No & $36(66.6)$ & $32(69.6)$ & \\
\hline \multicolumn{4}{|c|}{ ¿Cree que la vacuna sirva? } \\
\hline Sí & $43(79.6)$ & $37(80.4)$ & \multirow[t]{2}{*}{0.561} \\
\hline No & $11(20.4)$ & 9 (19.6) & \\
\hline \multicolumn{4}{|c|}{ ¿Está vacunado contra la influenza? } \\
\hline Sí & $18(33.4)$ & $22(47.8)$ & \multirow[t]{2}{*}{0.102} \\
\hline No & $36(66.6)$ & $24(52.2)$ & \\
\hline \multicolumn{4}{|c|}{ ¿Cree que las medidas recomendadas por el gobierno sirvan de algo? } \\
\hline Sí & $44(81.4)$ & $33(71.7)$ & \multirow[t]{2}{*}{0.190} \\
\hline No & $10(18.6)$ & $13(28.3)$ & \\
\hline \multicolumn{4}{|c|}{ ¿Cree que la información dada por los medios de comunicación es confiable? } \\
\hline Sí & $23(42.5)$ & 14 (30.4) & \multirow[t]{2}{*}{0.147} \\
\hline No & $31(57.5)$ & $32(69.6)$ & \\
\hline
\end{tabular}


Tabla 4: Comparación de la pregunta sobre la «técnica de rebaño».

¿Está usted de acuerdo con la técnica de infección de «rebaño» para acabar más rápido con la pandemia?

Respuestas, $\mathrm{n}(\%) \quad \mathrm{p}\left(\chi^{2}\right)$

\begin{tabular}{|c|c|c|c|}
\hline Hombre (Sí) & $8(14.9)$ & $46(85.1)$ & 0.028 \\
\hline Mujeres (No) & $1(2.2)$ & $45(97.8)$ & \\
\hline \multicolumn{4}{|c|}{ Hombres con nivel educativo de secundaria o menos } \\
\hline Sí & $7(24.2)$ & $22(75.8)$ & 0.002 \\
\hline No & $2(8)$ & $23(92)$ & \\
\hline \multicolumn{4}{|c|}{ Edad menor de 40 años } \\
\hline Sí & $10(33.4)$ & $20(66.6)$ & 0.183 \\
\hline No & $8(21.5)$ & $62(88.5)$ & \\
\hline \multicolumn{4}{|c|}{ Nivel educativo de secundaria o menos } \\
\hline Sí & $8(16.7)$ & $40(83.3)$ & 0.011 \\
\hline No & $1(2)$ & $51(98)$ & \\
\hline
\end{tabular}

de conspiración pueden fomentar un sentido de cognición aceptable cuando la situación carece de claridad, coherencia y mensaje of icial comprensible. ${ }^{26}$

En nuestro grupo, en los hombres con secundaria o menos son en quienes predominan más las creencias falsas, lo que puede traducirse en minoría o grupo vulnerable. En ese sentido, ya se han hecho estudios donde se evalúan los grupos vulnerables, encontrando que en los grupos más vulnerables es donde predominan las infecciones y la mortalidad, como el estudio de Millet que revela menor mortalidad entre pacientes de raza caucásica durante la pandemia de COVID 19.27

Nuestros resultados también aportan que más de la mitad no estaban vacunados en el momento de la entrevista (60\%) y 10\% no usaban cubrebocas antes de tener síntomas. Al evaluar su confianza, se encontró que hay más confianza en el gobierno que en los medios de comunicación en cuanto al manejo de la pandemia y de la información respectivamente. En ese mismo sentido, en estudios anteriores sobre vacunación en la pandemia de la influenza se observó que en voluntarios con niveles académicos bajos, la mayoría de entrevistados no se había vacunado contra la influenza y aunque ninguna pandemia es igual, se infiere que la posibilidad de vacunarse contra la COVID-19 es baja en este grupo. Mismos resultados reportaron Liao y colaboradores, sólo 7\% de 896 participantes refirieron que era probable que se fueran a vacunar y lo relacionaron con la baja percepción de la gravedad de la enfermedad. ${ }^{28} \mathrm{Y}$ en cuanto a la confianza, en el estudio de Lazarus y su equipo también fue evidente la gran variabilidad de respuestas en cuanto a la confianza en sus respectivos países, en algunos la confianza era alta y en otros muy baja, lo que se relacionaba con las medidas de contención emprendidas por dichos gobiernos. ${ }^{29}$

Por último y como David Singh interpreta el papel de la religión en la dispersión de la pandemia (2020) «los humanos somos una especie intensamente social, las creencias sobrenaturales están programadas en nuestro cerebro». Cierto tipo de fe es más universal que la creencia en lo sobrenatural. Como ocurre con las religiones, la mayoría de las formas de fe pueden ser interpretadas por los no creyentes como «no racional», pero esto no significa que sean necesariamente falsas. La fe permite a los humanos acercarse a cosas invisibles y también ofrece un medio para cooperar. Éste es también un factor básico en hacernos quienes somos: seres sociales y religiosos. ${ }^{30}$ 


\section{CONCLUSIONES}

En el presente trabajo un cuarto de los encuestados no creía en la existencia del virus SARS-COV-2, el cual se incrementaba con los voluntarios hombres de nivel educativo bajo. Asimismo, la mayoría no estaban vacunados contra la influenza y un tercio tenía la creencia de que se les extraía líquido de la rodilla a los pacientes y se les dejaba morir en los hospitales.

\section{REFERENCIAS}

1. Bouadma L, Lescure FX, Lucet JC, Yazdanpanah Y, Timsit JF. Severe SARS-CoV-2 infections: practical considerations and management strategy for intensivists. Intensive Care Med. 2020; 1-4. Available in: https://doi.org/10.1007/s00134020-05967-x

2. Ojha V, Mani A, Pandey NN, Sharma S, Kumar S. CT in coronavirus disease 2019 (COVID-19): a systematic review of chest CT findings in 4410 adult patients. EuropeanRadiology. 2020; 30: 6129-6138. Available in: https://link.springer.com/article/10.1007/s00330-02006975-7

3. Carrillo-Vega MF, Salinas-Escudero G, García-Peña C, Gutiérrez-Robledo LM, Parra-Rodríguez L. Early estimation of the risk factors for hospitalisation and mortality by COVID-19 in México. MedRxiv. 2020:1-18. Available in: https:// doi.org/10.1101/2020.05.11.20098145

4. Lobdell KW, Hariharan S, Smith W, Rose GA, Ferguson B, Fussell C. Improving health care leadership in the COVID-19 era. NEJM Catalyst. 2020: 1-7. doi: 10.1056/CAT.20-0225.

5. COVID-19 Tablero México-CONACYT-CentroGeo. Available in: https://datos.covid-19.conacyt.mx/

6. La OMS reconoce semáforo epidemiológico de México. Nota periodística. 14 de octubre del 2020. Disponible en: https://bajio.telediario.mx/nacional/oms-reconocesemaforo-epidemiologico-de-mexico

7. Urzúa A, Vera-Villarroel P, Caqueo-Urízar A, PolancoCarrasco R. La psicología en la prevención y manejo del COVID-19. Aportes desde la evidencia inicial. Terapia psicológica. 2020; 38 (1): 103-118. Disponible en: https://scielo.conicyt.cl/pdf/terpsicol/v38n7/0718-4808terpsicol-38-01-0103.pdf

8. Regi J, Narendran M, Bindu A, Beevi N, Benny M. Public perception and preparedness for the pandemic COVID 19: A Health Belief Model approach. Clinical Epidemiology and Clobal Health. 2027; 9: 41-46. Available in: https://doi. org/10.1016/j.cegh.2020.06.009

9. Shaoa W, Haob F. Confidence in political leaders can slant risk perceptions of COVID-19 in a highly polarized environment. Social Science \& Medicine. 2020; 261 : 113235. Available in: https://doi.org/10.1016/j.socscimed.2020.113235
10. Nilima N, Kaushikb S, Tiwaryc B, Kant-Pandeyc P. Psychosocial factors associated with the nationwide lockdown in India during COVID-19 pandemic. Clinical Epidemiology and Global Health. 2027; 9: 47-52. Available in: https://doi. org/10.1016/j.cegh.2020.06.010

11. Moreno-Casbas MT. Factores relacionados con el contagio por SARS-CoV-2 en profesionales de la salud en España. Proyecto SANICOVI. Enfermería Clínica. 2020; 30 (6): 360-370. Disponible en: https://doi.org/10.1016/j. enfcli.2020.05.027

12. Foley DA, Chew R, Raby E, Tong SY, Davis JS. COVID-19 in the pre-pandemic period: a survey of the time commitment and perceptions of infectious diseases physicians in Australia and New Zealand. Intern Med J. 2020; 50 (8): 924-930. doi: 10.1171/imj.14941.

13. Sanz-Almazán M, Rodríguez-Ledob P. Conocimiento y percepción de las medidas adoptadas frente a la COVID-19 por los profesionales de atención primaria al inicio de la pandemia. Med Gen Fam. 2020; 9 (3): 95-103. Disponible en: http://dx.doi.org/10.24038/mgyf.2020.023

14. Juárez-Huet N, Ramírez-Morales R, Olivas-Hernández O, Odgers-Ortiz O. Encuesta sobre coronavirus, bienestar y religiosidad (COBIRE 2020) Documentos de contingencia. El Colegio de la Frontera Norte (CONACYT) 2020:1-39. Disponible en: https://www.colef.mx/estudiosdeelcolef/ encuesta-sobre-coronavirus-bienestar-y-religiosidadcobire-2020/

15. Mejía CR, Quispe-Sancho A, Rodríguez-Alarcón JF, CasaValero L, Ponce-López VL, Varela-Villanueva ES et al. Factores asociados al fatalismo ante la COVID-19 en 20 ciudades del Perú en marzo 2020. Rev Haban Cienc Méd [Internet]. 2020[citado]; 19 (2): e_3233. Disponible en: www. revhabanera.sld.cu/index.php/rhab/article/view/3233/2496

16. Mizrahi D. Sociología del coronavirus: cuando la cultura de los países puede ser una ayuda o un obstáculo ante la pandemia. En: Dossier Covid-19 Impactos socioculturales de la pandemia. Centro de Estudios en Salud y Sociedad, Colegio de Sonora. 2020: 47-55. Disponible en: https:// www.colson.edu.mx/promocion/img/Dossier\%20Covid19_ Impactos\%20socioculturales.pdf

17. Gohel KH, Patela PB, Shaha PM, Patela JR, Panditb N, Rautc A. Knowledge and perceptions about COVID-19 among the medical and allied health science students in India: an online cross-sectional survey. Clinical Epidemiology and Global Health. 2021; 9: 104-109. Available in: https://doi. org/10.1016/j.cegh.2020.07.008

18. Raude J, Debin M, Souty C, Guerris C, Turbelin I et al. Are people excessively pessimistic about the risk of coronavirus infection? Scientific literature from EHESP School of Public Health. 2020: 1-6hal-02544148? doi: 10.31234/osf.io/364qj.

19. Morice A, Ávila-Agüero ML. Mitos, creencias y realidades sobre las vacunas. Acta Pediátr Costarric. 2008; 20 (2): 6065. Disponible en: https://www.scielo.sa.cr/pdf/apc/v20n2/ a07v20n2.pdf 
20. Fisher KA, Bloomstone SJ, Walder J, Crawford S, Fouayzi H, Mazor KM. Attitudes toward a potential SARS-CoV-2 vaccine: a survey of U.S. adults. Ann Intern Med. 2020; 173 (12): 964-973. doi: 10.7326/M20-3569.

21. Velayos JL, Sánchez RD. El apego en la práctica clínica durante la pandemia COVID-19. Revista de Psicoterapia. 2020; 31 (116): 295-309. Disponible en: https:// doi.org/10.33898/rdp.v31i176.391

22. Asimakopoulou K, Hoorens V, Speed E, Coulson NS, Antoniszczak D, Collyer F et al. Comparative optimism about infection and recovery from COVID-19; implications for adherence with lockdown advice. Health Expect. 2020; 23: 1502-1511. doi: 10.1171/hex.13134.

23. Escolà-Gascón A, Marín FX, Rusiñol J, Gallifa J. Pseudoscientific beliefs and psychopathological risks increase after COVID-19 social quarantine. Clobalization and Health. 2020; 16 (72): 1-11. Available in: https://doi. org/10.1186/s12992-020-00603-1

24. Sallam M, Dababseh D, Yaseen A, Al-Haidar A, Ababneh N, Bakri F et al. Conspiracy beliefs are associated with lower knowledge and higher anxiety levels regarding COVID-19 among Students at the University of Jordan. Int J Environ Res Public Health. 2020; 17: 4915. doi: 10.3390/ijerph17144915

25. Freeman D, Waite F, Rosebrock L, Petit A, Causier Ch, East A. Coronavirus conspiracy beliefs, mistrust, and compliance with government guidelines in England. Psychological Medicine. 2020;1-13. Available in: https://doi. org/10.1017/S0033291720001890

26. Panchal R, Jack A. The contagiousness of memes: containing the spread of COVID-19 conspiracy theories in a forensic psychiatric hospital. BJPsychBulletin. 2020; 1-7. doi: 10.1192/bjb.2020.120.

27. Millett CA. New pathogen, same disparities: why COVID-19 and HIV remain prevalent in U.S. communities of colour and implications for ending the HIV epidemic Journal of the International AIDS Society 2020, 23:e25639. Available in: https://doi.org/10.1002/jia2.25639

28. Liao Q, Cowling BJ, Lam WWT, Fielding R. Factors affecting intention to receive and self-reported receipt of 2009 pandemic (HTNT) Vaccine in Hong Kong: a longitudinal study. PLoS ONE. 2011; 6 (3): e17713. doi: 10.1371/journal. pone.0017713.

29. Lazarus JV, Ratzan S, Palayew A, Billari FC, Binagwaho A, Kimball $S$ et al. COVIDSCORE: A global survey to assess public perceptions of government responses to COVID-19 (COVID-SCORE-10). PLOS ONE 2020; 15 (10): e0240011. Available in: https://doi.org/10.1371/journal.pone.0240011

30. Singh DE. Role of Religions in the Spread of COVID-19. Journal of Ecumenical Studies. 2020; 55 (2): 289-310. Available in: https://muse.jhu.edu/article/760137

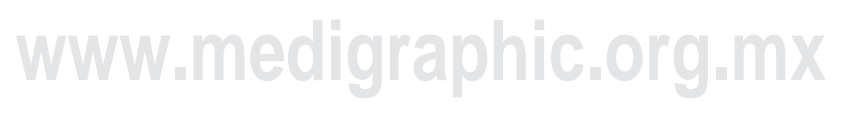

